\title{
PROFESSOR ANDRZEJ MATCZAK
}

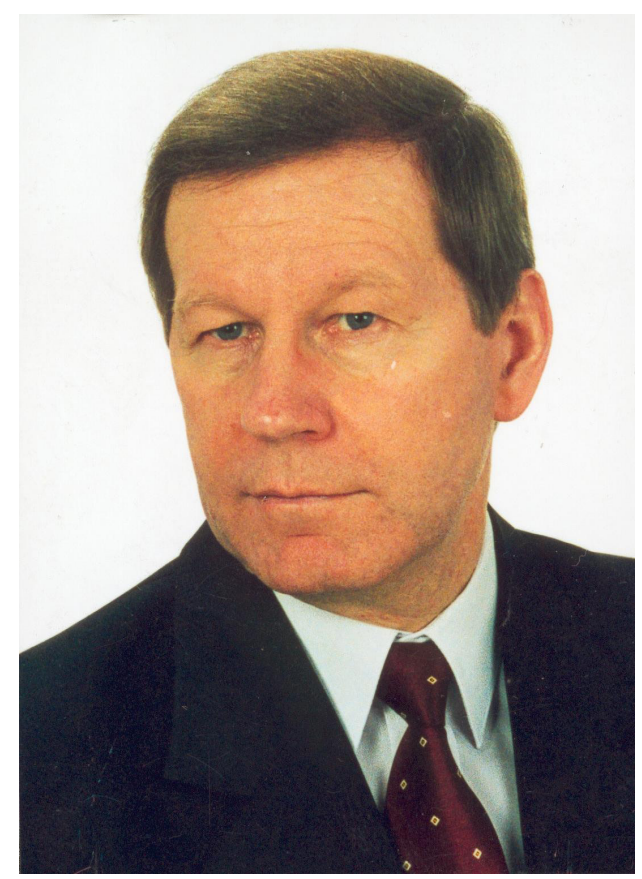

Prof. Andrzej Matczak is the Head of Geography of Hospitality Section, which is a part of the Institute of Urban Geography and Tourism Studies at the Faculty of Geographical Sciences, University of Lodz. He was born on $19^{\text {th }}$ May 1950 in Uniejów. The University of Lodz became a part of his life in 1968, when he started geographical studies at the Faculty of Biology and Earth Sciences. He wrote his MA thesis, entitled Changes in the forms of land use in the rural areas of Lodz urban agglomeration in 1963-1973 (based on census material provided by the Central Statistical Office), under the supervision of Prof. Tadeusz Olszewski. Initially, he worked at the Wojewódzka Pracownia Planów Regionalnych (Voivodeship Regional Planning Studio) in Lodz, but soon (1974) moved to the Economic Geography Section of the Institute of Geography at the University of Lodz whose head was Prof. Ludwik Straszewicz. In 1982, Andrzej Matczak defended his doctoral thesis, The recreational function of the Lodz suburban zone, prepared under the supervision of Prof. Stanisław Liszewski. He was granted his habilitation degree in 1992 for his work A model for tourism research: a methodological study, and the title of the full professor in 2002.

In 1995, Andrzej Matczak was appointed Director of the Institute of Economic Geography and Spatial Organization and, at the same time, Head of the Quantitative Methods in Geography Section at the Urban Geography and Tourism Studies Department, headed by Prof. Stanisław Liszewski. In 1996-1999, Prof. Matczak was the Vice-Dean of the Faculty of Biology and Earth Sciences at the University of Lodz. In 2001, the Faculty of Geographical Sciences was opened at the University of Lodz. As a result of the reorganization within the new department, Prof. Andrzej Matczak took up the position of Head of the Geography of Hospitality Section, which was initially a part of the Department, but in 2007 became a part of the Institute of Urban Geography and Tourism Studies. Andrzej Matczak worked at the University of Lodz as associate professor from 1997 until in 2004 when he was granted 
the title of full professor. During his working career, he cooperated with other universities as well: the Higher School of Tourism and Recreation in Warsaw (19982000), the Institute of Geography at Kazimierz Wielki University in Bydgoszcz (2001-2008), the Higher School of Humanities and Economics in Lodz (2003-2005) and the Faculty of Tourism and Geography at the Higher School of Economics in Bydgoszcz (2008-2012).

Prof. Andrzej Matczak has cooperated with various academic institutions in Lodz and in Poland. In 1995, he joined 'Lodz Society of Sciences and Arts' (Łódzkie Towarzystwo Naukowe). He is also an active member of the Polish Geographical Society, Lodz branch. In 1999-2003, he was the Secretary of the Geographical Sciences Committee at the Polish Academy of Sciences, and in 2016-2020, he was its active member.

Since 1992, Prof. Andrzej Matczak has been a member of the editorial team at the University of Lodz Publishing House, "Acta Universitatis Lodziensis. Folia Geographica Socio-Oeconomica", holding the position of Secretary in 1993-1994. Since 2000, he has been a member of the "Turyzm/Tourism" Editorial Council. He has taken part in numerous study visits and academic internships, both in Poland and abroad, e.g. in Leipzig (1975), Warsaw (1979-1980), Giessen (1983), Berlin (1985), Prague (1989, 1997), Sofia (1990), Tbilisi (1990), Aix-enProvence (1991), Prešov (1994), Bratislava (1999). In 1994, he received the Gold Badge of the University of Lodz and in 2001, he was presented with the "University of Lodz Service to Society and Science" medal, in 2002 with the Silver Cross of Merit. In 2019, the Faculty of Geography and Regional Studies at the University of Warsaw presented Prof. Andrzej Matczak with the Jerzy Kondracki Award. On 7th January 2008, during a special session of the town council, the decision was made to give Prof. Matczak the title of Honorary Citizen of the municipality of Uniejów.

The academic and research activity of Prof. Matczak is focused around two geographical sub-disciplines: tourism geography and settlement geography. Here are the most important research issues in the field of tourism geography which he has been involved with: methodology of research on tourism flows, transformations of the recreational and tourist functions in suburbs, the location and functioning of tourism enterprises, particularly hotels. Supplementary research has concerned the tourism function of cities and the geographical analysis of the tourism market. The research conducted by the Professor in the field of settlement geography covers the methodology of research concerning the spatial impact of cities by means of analysing the relationships between them and their surroundings, the functional and functionalspatial structure of cities, and the functional-spatial transformations of the suburban zone.
As his major achievement, Professor Andrzej Matczak evolved tourism research methodology. He described it in detail in his postdoctoral dissertation, which appeared in print. This work was an attempt to evaluate the earlier and to indicate the future directions in tourism research. What is more, Professor Andrzej Matczak made an attempt to adjust tourism research methodology to specific empirical conditions of protected (Matczak, 2002c), coastal (Matczak, 2005a, 2005c, 2009a) and urbanized areas (Matczak, 2012). He pointed to the relationship between tourism development and the perception of tourism space (Matczak, 1993a, 1994a, 1996a).

The discussion started by Prof. Andrzej Matczak regarding the recreational and tourist functions of suburban areas was a result of many years of empirical studies, mainly embracing the functional area of Lodz. First, he attempted to describe the tourist function in suburban zone (Matczak, 1984b, 1986a, 1986b, 1986c, 1986d, 1987a, 1987b, 1991a, 1991b, 1991c, 1994b, 1996b). Next, he concentrated on the analysis of tourism colonization processes in this zone (Matczak, 1995a, 1995b). He devoted his latest works on these issues to the pressure of the residential function in summer recreation areas in this zone (Matczak, 2005b, 2009b, 2016a). A summary of Prof. Andrzej Matczak's work on the tourism function is his review article entitled Research on the tourism function conducted at the tourism geography centre in Lodz (Matczak, 2015).

Prof. Andrzej Matczak initiated a discussion regarding the location of hotels, as well as the relevant research methodology (Matczak, 2004b; Matczak, Szkup, 2020). The most important works were the result of many years of studying the locations of facilities which provide hotel services in three urban centres: Lodz (Matczak, 1993b, 1998d, 2002b, 2004a, 2011c, 2016b, 2018, 2020); Bydgoszcz (Matczak, Grabiszewski, 2010); and Budapest (Matczak, Napierała, 2004, 2014); as well as on a national scale (Matczak, 2017). The works mentioned above concerned, above all, the development of hotel enterprises and their location policy during the socio-economic transformation in Central and East European countries. The results confirmed that the growth of the hotel industry sector depends on general economic development during the transformation.

The research conducted by Prof. Andrzej Matczak in the field of settlement geography was, above all, on the delimitation of urban region, based on an analysis of migration as well as the functional-spatial transformations of the urban regions under study. They were mostly case studies of small and medium-sized towns in Poland: Brodnica (Matczak, Szymańska, 1998; Szymańska, Matczak, 1996, 1998), Łask (Matczak, 2000b; Matczak, Michalski, 1999), Łęczyca (Matczak, 2002a), Prudnik (Matczak, 1984a), Sępólno Krajeńskie (Matczak, 
2011b), Sieradz (Matczak, 1998c; Matczak, Michalski, 1981) and Zduńska Wola (Matczak, 1998a, 1998b). Prof. Andrzej Matczak also published numerous comparative studies regarding these issues (Matczak, 1992, 2000a, 2011a; Matczak, Szymańska, 2000a, 2000b). The results of his research confirmed the particular character of the urbanization process in the functional regions of small and medium-sized towns, where departure from specialization towards the dominance of standard services was of key significance. The urbanization processes in the countries which went through systemic transformation were very different from the urbanization observed in West European countries (Szymańska, Matczak, 2002).

Prof. Andrzej Matczak has published 158 academic works, most of which are his own or co-authored articles or chapters in monographs presenting the results of empirical studies. He has been repeatedly asked to act as a reviewer in doctoral (17), postdoctoral (6) and professorial (4) procedures. He has successfully promoted eight doctoral candidates: Roman Szkup, Ewa Szafrańska, Krzysztof Parzych, Rafał Gotowski, Alicja Gonia, Tomasz Napierała, Andrzej Płoński and Monika Kozłowska-Adamczak. He has supervised 297 MA theses defended at the University of Lodz, 30 - at the Kazimierz Wielki University in Bydgoszcz and 17 - at the Higher School of Economics in Bydgoszcz. He has also supervised 50 BA diplomas at the Higher School of Tourism and Recreation in Warsaw and 51 at the Higher School of Humanities and Economics in Lodz.

Tomasz Napierała

The editors of „Turyzm/Tourism”, the employees of the Institute of Urban Geography and Tourism Studies, as well as the whole community of the Faculty of Geographical Sciences at the University of Lodz want to congratulate Professor Andrzej Matczak on his $70^{\text {th }}$ Birthday Anniversary, wishing him health, a lot of joy and prosperity, as well as further successes in his scientific career. Dear Professor, ad multos annos!

\section{REFERENCES}

Matczak, A. (1984a). Próba określenia zasięgu oddziaływania Prudnika na podstawie danych o przewozach towarowych. Acta Universitatin Lodzinensis. Folia Geographica, 3, 87-97.

Matczak, A. (1984b). Próba wykorzystania analizy morfologicznej do określenia funkcji wypoczynkowej osiedli w strefie podmiejskiej Łodzi. Problemy Turystyki, 2, 93-97.

Matczak, A. (1986a). Budownictwo letniskowe w strefie podmiejskiej Łodzi. Acta Universitatin Lodzinensis. Folia Geographica, 7, 137-166.
Matczak, A. (1986b). Dostępność komunikacyjna terenów wypoczynkowych w strefie podmiejskiej Łodzi. Przegląd Ekonomiczno-Społeczny m. Łodzi, 9, 113-125.

Matczak, A. (1986c). La fonction recreative de la zone suburbaine de la ville de Łódź. In: F. Vetter (ed.), Grosstadttourismus - Tourisme des grandes villes (pp. 304-315). Berlin: Union Géographique Internationale, Commission de Géographie du Tourisme et des Loisirs.

Matczak, A. (1986d). Przyrodnicze podstawy organizacji wypoczynku w strefie podmiejskiej Łodzi. Acta Universitatis Lodziensis. Turyzm, 2, 25-45.

Matczak, A. (1987a). Próba określenia funkcji wypoczynkowej osiedli podmiejskich na przykładzie Kolumny. Acta Universitatis Lodziensis. Turyzm, 3, 55-85.

Matczak, A. (1987b). Ruch wypoczynkowy w strefie podmiejskiej Łodzi. Acta Universitatin Lodzinensis. Folia Geographica, 8, 21-39.

Matczak, A. (1991a). Funkcja wypoczynkowa osiedli podłódzkich w świetle badań użytkowania ziemi. Acta Universitatis Lodzinensis. Folia Geographica, 13, 129-140.

Matczak, A. (1991b). Methods of studying the recreational function of suburban settlements. Zeszyty Naukowe Uniwersytetu Jagiellońskiego. Prace Geograficzne, 86, 109-122.

Matczak, A. (1991c). Przemiany przestrzenne wsi położonych w strefie podmiejskiej Łodzi na przykładzie rejonu Grotnik. Acta Universitatin Lodzinensis. Folia Geographica, 14, 33-46.

Matczak, A. (1992). Zmiany w strukturze funkcjonalnej miast Polski w latach 1973-1983. Acta Universitatin Lodzinensis. Folia Geographica, 17, 9-25.

Matczak, A. (1993a). Percepcja przestrzeni turystycznej Polski przez młodzież licealną Łodzi. Turyzm, 3 (2), 61-70.

Matczak, A. (1993b). Turystyczna baza noclegowa Łodzi i jej wykorzystanie w latach 1979-1990. Kronika m. Łodzi, 2, 51-67.

Matczak, A. (1994a). Percepcja przestrzeni turystycznej Polski przez zamiejscową młodzież akademicką studiującą w Łodzi. Turyzm, 4 (1), 23-33.

Matczak, A. (1994b). Turystyka i wypoczynek jako czynnik integracji i rozwoju regionu łódzkiego. In: A. Matczak (ed.), Studium wiedzy o regionie łódzkim. Wybrane problemy (pp. 161188). Lodz: Łódzkie Towarzystwo Naukowe.

Matczak, A. (1995a). Kolonizacja turystyczna obszarów wiejskich w strefie podmiejskiej Łodzi. In: A. Kowalczyk (ed.), Zmiany w przestrzeni geograficznej w warunkach transformacji społeczno-ekonomicznej (na przykładzie obszarów wiejskich) (pp. 49-59). Warsaw: Wydział Geografii i Studiów Regionalnych Uniwersytetu Warszawskiego.

Matczak, A. (1995b). Kolonizacja turystyczna strefy podmiejskiej Łodzi. Kronika m. Łodzi, 1, 108-119.

Matczak, A. (1996a). Percepcja regionalnej przestrzeni turystycznej przez uczniów szkół licealnych Łodzi. Turyzm, 6 (1), 63-75.

Matczak, A. (1996b). Turystyka i wypoczynek w łódzkim regionie miejskim. In: W. Michalski (ed.), Transformacja społecznogospodarcza Łodzi na tle regionu (pp. 99-109). Lodz: Centralny Urząd Planowania - Biuro Planowania Regionalnego w Łodzi, Polskie Towarzystwo Geograficzne - Oddział w Łodzi.

Matczak, A. (1998a). Delimitacja granic regionu miejskiego Zduńskiej Woli na podstawie danych o migracjach stałych. In: D. Szymańska (ed.), Ruchliwość przestrzenna ludności w okresie przemian ustrojowych (pp. 291-312). Toruń: Wydawnictwo Uniwersytetu Mikołaja Kopernika w Toruniu.

Matczak, A. (1998b). Delimitation of borders of the Zduńska Wola urban area using data on permanent migrations. Polish Population Review, 13, 191-210. 
Matczak, A. (1998c). Migracje jako element delimitacji regionu społeczno-ekonomicznego. Przykład Sieradza. Acta Universitatis Wratislaviensis. Studia Geograficzne, 2048, 93-110.

Matczak, A. (1998d). Turystyczna baza noclegowa Łodzi i jej wykorzystanie w latach 1979-1997. Turyzm, 8 (2), 27-56.

Matczak, A. (2000a). Delimitacja granic regionów miejskich: Łasku, Zduńskiej Woli i Sieradza na podstawie danych o migracjach stałych. In: D. Szymańska (ed.), Procesy i formy ruchliwości przestrzennej ludności w okresie przemian ustrojowych (pp. 37-56). Toruń: Wydawnictwo Naukowe Uniwersytetu Mikołaja Kopernika.

Matczak, A. (2000b). Delimitacja regionu społeczno-ekonomicznego małego miasta powiatowego w Polsce na podstawie danych o migracjach stałych. Przykład Łasku. Urbanne a Krajinne Studie, 3, 248-261.

Matczak, A. (2002a). Delimitacja zaplecza społeczno-ekonomicznego Łęczycy. In: J. Słodczyk (ed.), Przemiany bazy ekonomicznej i struktury przestrzennej miast (pp. 205-218). Opole: Wydawnictwo Uniwersytetu Opolskiego.

Matczak, A. (2002b). Efektywność wykorzystania rejestrowanych obiektów noclegowych w Łodzi w latach 1979-2000. Turyzm/ Tourism, 12 (2), 65-78.

Matczak, A. (2002c). Metodyka badań ruchu turystycznego na obszarach chronionych. In: J. Partyka (ed.), Użytkowanie turystyczne parków narodowych. Ruch turystyczny - zagospodarowanie - konflikty-zagrożenia (pp. 17-22). Ojców: Ojcowski Park Narodowy.

Matczak, A. (2004a). Hotelarstwo polskie w okresie przemian. Przykład Łodzi. Zeszyty Naukowe WSG w Bydgoszczy. Seria Turystyka i Rekreacja, 1, 21-34.

Matczak, A. (2004b). Metodologia badań nad lokalizacją hoteli. In: A. Matczak (eds), Lokalizacja hoteli w krajowych metropoliach Europy Środkowo-Wschodniej (pp. 11-43). Lodz: Łódzkie Towarzystwo Naukowe.

Matczak, A. (2005a). Badania przyjazdowego ruchu turystycznego w rejonie Ustki. In: E. Rydz (ed.), Ksztattowanie funkcji turystycznych w miejscowościach uzdrowiskowych (pp. 265-284). Słupsk: Wydawnictwo Pomorskiej Akademii Pedagogicznej w Słupsku.

Matczak, A. (2005b). Rozwój podmiejskich osiedli letniskowych w świetle koncepcji cyklu życia obszaru turystycznego. Przykład podłódzkiego letniska w Wiśniowej Górze. In: B. Domański, S. Skiba (eds), Geografia i sacrum (pp. 265284). Kraków: Instytut Geografii i Gospodarki Przestrzennej UJ w Krakowie.

Matczak, A. (2005c). Turystyka w przestrzeni miejskiej Ustki - polskiego kurortu nadbałtyckiego. Turyzm/Tourism, 15 (1-2), 139-149.

Matczak, A. (2009a). Ruch turystyczny w Rowach w latach 1999-2007. In: R. Muszkieta, M. Napierała (eds), Wspomaganie rozwoju i edukacji człowieka poprzez turystyke, sport i rekreacje (pp. 25-47). Bydgoszcz: Wydawnictwo WSG w Bydgoszczy.

Matczak, A. (2009b). Urbanizacja podmiejskich osiedli letniskowych. Przykład Wiśniowej Góry w strefie podmiejskiej Łodzi. Turyzm/Tourism, 19 (1-2), 67-72.

Matczak, A. (2011a). Changes in the functional struckture of the cities in the Lodz region in 1973-2007. In: A. Runge, A. Kuczabski (eds), Medium-sized towns of Central-Eastern Europe in the period of economic system transportation and social changes (pp. 35-44). Kharkiv: Publishing House ADNDU.

Matczak, A. (2011b). Identyfikacja regionu społeczno-ekonomicznego małego miasta. Przykład Sępólno Krajeńskie. In: K. Marciniak, R. Sikora, D. Sokołowski (eds), Koncepcje i problemy badawcze geografii. Profesorowi Wiesławowi Maikowi w 70-lecie urodzin (pp. 23-28). Bydgoszcz: Wydawnictwo WSG w Bydgoszczy.
Matczak, A. (2011c). Turystyczna baza noclegowa w Łodzi w latach 1979-2009. In: B. Włodarczyk (ed.), Turystyka. Księga jubileuszowa w 70. rocznice urodzin Profesora Stanisława Liszewskiego (pp. 287-296). Lodz: Wydawnictwo Uniwersytetu Łódzkiego.

Matczak, A. (2012). Koncepcja monitorowania ruchu turystycznego w dużym mieście. In: S. Sitek (ed.), ",Stare i nowe" problemy badawcze geografii społeczno-ekonomicznej (pp. 77-91). Sosnowiec: Polskie Towarzystwo Geograficzne, Oddział Katowicki, Uniwersytet Śląski, Wydział Nauk o Ziemi.

Matczak, A. (2015). Funkcja turystyczna w badaniach łódzkiego ośrodka geografii turyzmu. In: Z. Młynarczyk, A. Zajadacz (eds), Uwarunkowania i plany rozwoju turystyki. Vol. XIV: Rozwój badań geograficznych nad turystyka (pp. 101-116). Poznań: Uniwersytet im. Adama Mickiewicza w Poznaniu.

Matczak, A. (2016a). Urbanizacja podłódzkich osiedli letniskowych. Przykład Miasta-Lasu Kolumny. In: A. Zborowski (ed.), Człowiek, społeczeństwo, przestrzeń (pp. 105121). Myczkowce-Kraków: Instytut Geografii i Gospodarki Przestrzennej UJ w Krakowie, Centrum Kultury Ekumenicznej.

Matczak, A. (2016b). Zmiany w wielkości i strukturze turystycznej bazy noclegowej Łodzi po 1989 r. In: D. Sokołowski, P. Tomczykowska (eds), Kreatywność w turystyce. Innowacyjne rozwiazania we wspótczesnej turystyce (pp. 11-29). Toruń: Wydawnictwo Naukowe Uniwersytetu Mikołaja Kopernika.

Matczak, A. (2017). Zmiany w liczbie i pojemności hoteli w Polsce. Turyzm/Tourism, 27 (2), 77-87. DOI: https://doi. org/10.18778/0867-5856.27.2.07

Matczak, A. (2018). Zmiany w potencjale i wykorzystaniu hoteli łódzkich po 1989 r. In: S. Sitek (ed.), "Stare i nowe" problemy badawcze geografii społeczno-ekonomicznej (pp. 7-27). Sosnowiec: Polskie Towarzystwo Geograficzne, Oddział Katowicki, Uniwersytet Śląski, Wydział Nauk o Ziemi.

Matczak, A. (2020). Zmiany w wielkości i użytkowaniu hoteli w Łodzi. In: R. Szkup (ed.), Turystyczna baza noclegowa województwa łódzkiego (pp. 127-147). Lodz: Wydawnictwo Uniwersytetu Łódzkiego. DOI: https://doi.org/10.18778/8142-645-9.05

Matczak, A., Grabiszewski, M. (2010). Turystyczna baza noclegowa Bydgoszczy i jej wykorzystanie w latach 1979-2009. Zeszyty Naukowe WSG w Bydgoszczy. Seria Turystyka i Rekreacja, $15,105-131$

Matczak, A., Michalski, W. (1981). Przepływy towarowe jako podstawa wyznaczania związków przestrzennych Sieradza. Acta Universitatin Lodzinensis. Folia Geographica, 1, 129-152.

Matczak, A., Michalski, W. (1999). Zmiany w zasięgu przestrzennym oddziaływania społeczno-ekonomicznego Łasku w okresie transformacji ustrojowej. In: A. Matczak (ed.), Studia nad struktura funkcjonalno-przestrzenna miasta. Przykład Łasku (pp. 77-98). Lodz: Wydawnictwo Uniwersytetu Łódzkiego.

Matczak, A., Napierała, T. (2004). Lokalizacja hoteli w Budapeszcie. In: A. Matczak (ed.), Lokalizacja hoteli w krajowych metropoliach Europy Środkowo-Wschodniej (pp. 83-100). Lodz: Łódzkie Towarzystwo Naukowe.

Matczak, A., Napierała, T. (2014). Zmiany w lokalizacji hoteli w Budapeszcie. In: G. Gołembski, A. Niezgoda (eds), Turystyka wobec zmian wspótczesnego świata. Zmiany, bariery, innowacje (pp. 127-139). Poznań: Wydawnictwo Uniwersytetu Ekonomicznego w Poznaniu.

Matczak, A., Szkup, R. (2020). Turystyczna baza noclegowa jako przedmiot analiz naukowych. In: R. Szkup (ed.), Turystyczna baza noclegowa województwa łódzkiego (pp. 9-37). 
Lodz: Wydawnictwo Uniwersytetu Łódzkiego. DOI: https:// doi.org/10.18778/8142-645-9.01

Matczak, A., Szymańska, D. (1998). Permanent migrations as a measure of town's impact (the Brodnica case). Polish Population Review, 13, 175-190.

Matczak, A., Szymańska, D. (2000a). Baza ekonomiczna małych miast powiatowych $\mathrm{w}$ świetle pomiarów bezpośrednich. Studium porównawcze Brodnicy i Łasku. In: J. Słodczyk (ed.), Społeczne, gospodarcze i przestrzenne przeobrażenia miast (pp. 95113). Opole: Wydawnictwo Uniwersytetu Opolskiego.

Matczak, A., Szymańska, D. (2000b). Identyfikacja i pomiar bazy ekonomicznej miasta na przykładzie Brodnicy i Łasku. Acta Universitatis Nicolai Copernici. Geografia, 104, 191-211.
Szymańska, D., Matczak, A. (1996). Ruch wędrówkowy ludności i jego zasięg oddziaływania na przykładzie miasta Brodnicy. In: J. Falkowski (ed.), Wybrane problemy geografii społecznoekonomicznej i zagospodarowania przestrzennego (pp. 177-187) Toruń: Wydawnictwo Uniwersytetu Mikołaja Kopernika w Toruniu.

Szymańska, D., Matczak, A. (1998). Region miejski Brodnicy w świetle migracji stałych. Acta Universitatis Wratislaviensis. Studia Geograficzne, 2048, 79-91.

Szymańska, D., Matczak, A. (2002). Urbanization in Poland: Tendencies and transformation. European Urban and Regional Studies, 9 (1), 39-46. DOI: https://doi. org/10.1177/096977640200900104 